Designing individualisation of eco information: A conceptual framework and design toolkit

Corresponding author:

Sze Yin Kwok

Department of Design, Brunel University London, Uxbridge, Middlesex, UK

Sze.Kwok@brunel.ac.uk

Tel: 07803430026

Co-authors:

Professor David Harrison

Department of Design, Brunel University London, Uxbridge, Middlesex, UK

David.Harrison@brunel.ac.uk

Dr Alessio Malizia

Department of Computer Science, Brunel University London, Uxbridge, Middlesex, UK alessio.malizia@brunel.ac.uk 


\title{
Designing individualisation of eco information: A conceptual framework and design toolkit
}

\author{
Abstract \\ Eco labelling is the practice of eco information provision that most directly addresses \\ consumer behaviour. Nowadays consumers are facing difficulties in perceiving and \\ understanding existing eco labels. In previous work we proposed the conceptual \\ framework of eco information individualisation which tailors eco labels according to \\ the specific needs of individual users using contextual technologies. This paper extends \\ the conceptual framework by introducing a more structured way of considering the \\ personal data and product data requirements, and reports the development of a design \\ toolkit that aims to support designers in the designing of individualised eco \\ information. A design workshop was carried out to introduce the concept to designers, \\ and evaluate the usability and usefulness of the toolkit. Positive responses were \\ received. The design outputs generated from the workshop were considered largely \\ feasible and have the potential to be developed into digital prototypes. These indicate \\ that it is possible for designers to learn to design eco information individualisation in a \\ short time. This paper is a step towards a greater understanding of designing \\ individualised eco information.
}

Keywords: eco labelling; information individualisation; user centred design; human computer interaction; sustainable consumer behaviour

\section{Introduction}

The provision of eco information is one potential design intervention strategy to encourage more sustainable consumer behaviour (Bhamra, Lilley, and Tang 2011; Froehlich 2011). Among existing eco information tools, eco labelling addresses consumer behaviour most directly (Kwok, Harrison, and Qin 2013). However literature suggests that consumers are having difficulties in perceiving, understanding and trusting eco labels (Cohen and Vandenbergh 2012; Thøgersen 2002). Numerous studies show that existing eco labels fail, at least partly, to provide sufficient information and to communicate with consumers effectively (van Amstel, Driessen, and Glasbergen 2008; Hartikainen et al. 2013; Upham, Dendler, and Bleda 2011). 
During an initial report of this research work (Kwok, Harrison, and Qin 2014), we discussed the opportunities provided by contextual technology, a collective term for various technologies enabling the 'age of context' (Scoble and Israel 2014), to improve current eco labelling. We proposed the concept of 'eco information individualisation' which tailors eco labels according to the specific needs of the individual user. With technologies embedded on the product and the user, both of them can act as data carriers, have a traceable record, a 'life history', and exchange information digitally. This 'life history' is a record of the context embracing the past (e.g. shopping history of user, usage behaviour of user, logistic record of product), the present status (e.g. situation of user, product condition) and perhaps the future (e.g. personal goal of the user, environmental performances associated with different product disposal methods). An enabled product can be intelligent enough to appeal to the particular preferences of a user. This can potentially change people's perception and understanding towards everyday products and impact consumer decision-making processes (Kwok, Harrison, and Qin 2014).

Figure 1 shows the refined conceptual framework. There are four main components, namely personal data, product data, technology on user, technology on product. Personal data can be stored with technology on the user. Product data can be either stored locally or remotely in a cloud archive and be accessed by technology on the product. Contextual technologies can enable digital interaction between product and user, and result in individualised eco information feedback. More details about this concept and an initial description of this framework are described in our earlier conference paper (ibid.).

\section{Methodology}

To provide further information regarding the specific personal context, product context and enabling technologies for designing eco information individualisation, this paper extends the work of the above paper (ibid.) by: 
- Introducing a more structured way of considering the personal data and product data requirements for this proposed system;

- Reporting on the development of a design toolkit to support designers in the designing of individualised eco information;

- Describing a workshop carried out with designers to evaluate the usability and usefulness of the toolkit.

\section{Eco information individualisation design toolkit: design and development}

\subsection{Background}

In the earlier paper (ibid.), we reported outcomes of a focus group study that served as a preliminary evaluation of the conceptual framework. Ten participants took part in the two semi-structured focus group interviews. The designers who took part in the focus groups expressed positive comments about the conceptual framework, and discussed the role of user centred design in designing eco information individualisation. Some of them asked for more details of the framework. This led to the idea of developing a design toolkit that aims to help designers to understand the concept of eco information individualisation, support problem framing and stimulate design ideas.

Card-based tools have previously been used as a presentation format to put together knowledge from diverse areas into a re-usable form to stimulate designers’ thinking. Relevant examples include the Design with Intent Tool (Lockton, Harrison, and Stanton 2010), IDEO Method Cards (IDEO 2003), the Human-Centred Design Toolkit (IDEO 2015), the CoLab Design Tool (Pei 2009) and Drivers of Change card sets (Arup 2009).

\subsection{Toolkit structure}

The four major components needed to produce individualised eco labels were personal data, product data, technology on user and technology on product. This information was used to drive the content structure of the eco information individualisation design toolkit. 
The toolkit consisted of a short guidebook that introduces the concept of eco information individualisation, design templates that assist with structuring label design and picturing the use of technology, and 25 cards that provide structured information on user context, product context and technologies in relation to eco information individualisation.

\subsubsection{Person cards}

To structure the personal data element, we extended the user context model of Kofod-Petersen \& Aamodt (2003) to provide a model for personal data aspects of eco information individualisation. There were five key aspects to this personal context model, namely personal profile, task context, spatio-temporal context, past history and social context. The personal profile was further divided into mental context and physiological context.

Building on this context model, eight person cards were designed to provide information about potential dimensions of individualisation around people's data, with respect to their cognitive styles ('verbaliser vs imager' and 'wholist vs analytic'), 'preference \& interest', 'physiological factors', 'time \& location', 'task related behaviour', 'history \& habit' and 'social factors'.

\subsubsection{Product cards}

A card sorting study was conducted to uncover how users make sense of existing eco labels. The study involved nine participants in six card sorts, which included five individual sorts and one team sort with four participants. The appropriate sample size for card sorting depends on the purpose of the study. As this card sorting study only intended to gather some broad ideas of how people categorise eco labels and how people perceive the information displayed on eco labels, and a complementary user research method (interview) was used, a small number of card sorts was deemed enough. Both individual and team card sorts were conducted, for the advantages of collecting rich insights from discussion in a team sort and collecting more data from fewer participants. The eligibility criteria used for participants selection were rather simple: i) they had to be end users of eco labels, who basically could be anyone with everyday shopping experience; ii) they were required to have a good command 
of English, so that their use of vocabularies made sense. The recruited participants had varied demographic characteristics, in terms of age, gender, profession and nationality. The results of this study provided insights for a possible organisation scheme which groups eco information by information formats and by eco information content. The latter can further be divided into 4 categories: product categories, life cycle stages, eco attributes and issuing organisation.

Informed by the organisation scheme identified in the card sorting study, eight product cards were designed. Four of these cards describe relevant product eco information content: i) 'product category' that determines the shopping context; ii) 'issuing organisation' which affects consumer's trust and confidence; iii) 'life cycle analysis' that results in an inventory of environmental impact throughout various product life cycle stages; and iv) the wide variety of 'eco attributes' that represents various product qualities. The other four cards provide insights about presentation formats of eco information on products, namely i) using 'colour' to convey meaning; ii) tips on 'data visualisation'; iii) considerations on the 'level of detail'; and iv) using 'richer medium' to provoke engagement.

\subsubsection{Technology cards}

A review of literature was conducted to identify the opportunities for individualising eco labels enabled by emerging contextual technologies. Findings from the literature were used to inform the design of the 'technology' card deck. This card deck reflects two components of the conceptual framework, namely technology on user and technology on product. This deck contains nine technology cards to provide information about useful technologies that enable eco information individualisation. Topics covered include 'barcode \& QR code', 'augmented reality', 'iBeacon', 'RfID \& NFC', 'mobile \& wearables', 'social media', 'the Internet of Things', 'location-based service' and 'cloud computing'. Table 1 lists all cards in the toolkit.

\subsection{Details of card and template design}

All cards are in A6 size with contents printed on both sides. On the front is a big image which acts as a visual aid to illustrate the overall concept of the card concisely and clearly. The images were chosen 
to serve one or more of the following purposes: i) reinforce the messages of the card with emotive and playful visual elements; ii) present the concepts through metaphor; iii) depict explicitly the scenarios/ examples suggested by the card contents . On the back are 4 items - title, sub title, main text and an interesting fact supplemented with an explanatory image. Figure 2 shows an example of the person card.

The idea of information individualisation is rooted in the fact that context around user and product can be very complex and the amount of contextual data captured can be enormous. When visualising these data in the form of an eco label, there may exist many possible dimensions for tailoring - cognitive styles, time, location, social parameters, parameters of environmental impact, size of fonts, visual capacity or impairment of user and conditions of viewing, to name a few. Each dimension consists of a continuum of label designs, so that individuals with different needs would see different labels accordingly. For example, when tailoring upon the dimension of 'level of detail', designers should create a continuum of labels with different levels of detail in order to satisfy different individuals. Figure 3 shows a design template created to help position 9 design variations in the form of a $3 \mathrm{X} 3$ matrix. This template provides a basic structure for 2-dimensional eco information individualisation, with 3-levels of variation on each dimension. Examples of the template usage can be seen in Figure 7 and Figure 9.

\subsection{Eco information designer workshop}

\subsection{Introduction}

A design workshop was held to introduce the concept of eco information individualisation and the design toolkit to participating designers. The session also served as an evaluation of the design toolkit. Three main research questions were asked:

(1) Is it possible for a designer to understand the idea of eco information individualisation in the workshop with the aid of the design toolkit?

(2) Were feasible concepts generated? 
(3) How did the designers feel about the toolkit in terms of usability and usefulness?

\subsection{Method}

\subsubsection{Participants}

Workshops typically tend to involve 8 to 20 people working on carefully planned activities to generate specified outputs (RSSB 2008). The workshop reported in this paper involved two facilitators and eleven designer participants. All designers had knowledge of eco design, graphic design and an understanding of life cycle analysis. Nine of them had industrial experience ranging from 1 year to 15 years long.

\subsubsection{Procedure}

The workshop lasted for three hours. At the beginning of the workshop, the designers were introduced to the background of Eco Information Individualisation, then they were asked to work on four 40 minutes long design activities. A 5-minute break was given after every task, while the facilitator distributed materials and explained the next task. We are aware that fatigue issue might potentially happen after 3 hours of work. This might have a negative impact on the performance of the designers. But the results seem encouraging in that the quality of their designs were better in the later stage and did not appear to suffer after three hours. Also 3-hour long design sessions are acceptable in design practice.

The four activities were named 'Task 1', 'Task 2.1', 'Task 2.2' and 'Task 3' and were all individual activities. The designers were asked to design for the same brief throughout the workshop. The brief included a product specification, which contained eco information of various aspects for a bottle of milk, and four personas (Figure 4), which described individuals with different backgrounds, cognitive styles, values, personal goals, and shopping habits.

Task 1 was an unguided activity. The designers were not shown the design toolkit cards. Each designer had to choose to design for at least two of the personas and to design individualised eco 
labels for them. The reason for having to choose at least two personas was to inspire the designers to consider the needs of more than one individual, and so to encourage the generation of a range of designs.

Task 2.1 required the designers to design individualised eco labels for the same personas they chose previously with considerations of the personas' cognitive styles. Cognitive styles are the ways in which different people characteristically approach different cognitive tasks. Riding (1991) proposes there are two dimensions of cognitive styles, namely the Verbal-Imagery (VI) dimension and the Wholist-Analytic (WA) dimension. Before the task began, the designers were introduced to the two cards about cognitive styles from the 'person card' deck. For Task 2.2, the designers were asked to move their design ideas onto the 3X3 matrix design template (Figure 3) and structure their ideas on the axes representing the two dimensions of cognitive styles.

For Task 3, all cards from the toolkit were given to the designers. They were also given another 3X3 matrix design template (Figure 3), but this time the axes on the template were not labelled. The designers had the freedom to design for any user needs, and make their own choice of axes. They were also asked to mark which cards from the toolkit inspired their design ideas, (if any).

At the end of the workshop, the designers answered a post-task questionnaire that consisted of 17 questions related to the usability and usefulness of the design toolkit and two open-ended questions about their opinions.

\subsection{Results}

To address the research questions, we analysed three types of data collected from the workshop, which were i) observation during workshop, ii) design outputs from workshop, and iii) responses from the questionnaire.

\subsubsection{Task 1-Unguided design task}

We observed the following from the unguided activity and the design outputs from the session: 
- The design processes of the participating designers were diverse and visually and conceptually messy;

- Before starting to sketch the labels, many designers ideated the context, for instance, considering the personas’ core values, styles, personalities, desired functions;

- Most designers came up with two label designs in the shape of a symbol, one for each persona they chose;

- They presented on their label designs the information that the personas were interested in, for example the labels showed carbon footprint for Ben, and showed animal welfare for Lucy;

- Other common features in the design outputs included comparison of environmental performance between people or products, and integration of several eco attributes in one label;

Figure 5 shows a selection of sketches from Task 1 . These were produced by the participants P1, P4 and P10. Both P1 and P4 were designing around the personas Ben and Lucy; while P10 worked around the personas Ben and Jeff.

\subsubsection{Task 2-Designing eco information individualisation for cognitive styles}

Two cards from the toolkit were introduced to the designers before the design activity began. Design outputs from Task 2 showed that most of the designers had gained a deeper understanding of the concept of eco information individualisation by integrating spectra of design. They were able to individualise eco labels according to the personas' cognitive styles, and they showed considerations about the technologies used. The authors believed this was because the designers had learnt more from the toolkit about individualising eco labels to adapt to the cognitive styles of different personas. However it was also possible that the improvement of design outputs was caused by a practice effect the designers might have become more familiar with the task and more skilful over time.

Figure 6 shows the selected design outputs for Task 2.1 created by the participants P1, P4 and P10, who were all designing for the personas Ben and Lucy. As defined in the brief, Ben was a 
verbaliser and analytic. He was strong on numerical thinking, was concerned about carbon emission and was tech savvy. Lucy was an imager and wholist. She was fond of nature and animals, and she kept the use of technology to minimum.

To illustrate the two spectrums of cognitive styles, P1 and P4 aligned their label designs to two axes. P10 created separate designs for Ben and Lucy, but these were not design variations that could be aligned on a spectrum. Both P1 and P4 made use of QR code in their designs, while P10's design idea used augmented reality technology to overlay graphics on product packages (Figure 6).

Compared to Task 2.1, in Task 2.2 the designers better structured their design concepts using the given template (See Figure 7). Most designers had created two continuums of label design that accorded to the two dimensions of cognitive styles. On their design outputs, we could see a horizontal transformation from text-oriented designs to image-based designs, as well as a vertical transformation from holistic to analytic information presentation.

In Figure 7 we saw an example design generated by P4, who had designed nine labels that morphed into one another across the spectra. (Please note, as indicated by P4 on the sketch, the label in the middle of the top row should be swapped with the label on the right in the middle row.) On the top row were labels showing eco information holistically. On the top left was one label design with a number presenting the overall carbon emission of the product; on the top right was one label design that differentiated environmental performance of product using different colours, with green indicating more desired performance and red indicating less desired performance. In the bottom row were three designs that contained a more detailed breakdown of eco information, again on the left the design was relatively text based, to suit the need of a verbaliser, and on the right the presentation was more graphical, differentiating meanings using colours. The designs in the middle balanced the characteristics of both ends.

Apart from drawing the graphical labels, the designers also considered user scenarios. On the provided worksheet (part of the toolkit), they illustrated the technologies to be used to display the labels (Figure 8). 


\subsubsection{Task 3-Designing eco information individualisation for any chosen dimensions}

Provided with all cards from the design toolkit, the designers were able to think of many new ideas of eco information individualisation around various types of user and product contexts.

Figure 9 shows two examples created by P11 and P10 from Task 3. P11 was designing eco information with various levels of detail. The label on the left represented environmental performance by the amount of green colour filled on a leaf symbol. The label on the right, in addition to colouring the leaf symbol, showed detailed factors related to product environmental performance. P10 designed an individualised label that varied along two dimensions. The horizontal axis was related to how much time (how fast) the user had for reading the label. The vertical axis was related to how graphical the label was.

The designers were also asked to mark the cards that triggered ideas from their own thinking. The results are summarised in Figure 10. Twenty three cards, out of a total of twenty five, were tagged on the design outputs Task 3. The most popular card was 'level of detail' which appeared five times. This confirmed that the toolkit was able to enhance brainstorming and trigger new ideas.

\subsubsection{Feedback on the toolkit and the design workshop}

All designers appeared to be enthusiastic during the workshop, working quickly and giving positive verbal comments as they worked. It was observed that some designers were more able to pick up the framework than others. In particular there was one undergraduate designer who did not understand the framework and design task until the last exercise (Task 3). More experienced designers (with more working experience/ more senior level students) understood the framework in shorter time and created more 'thoughtful' designs. Their designs were more developed, detailed and logically sound.

In the post-task questionnaire, the participants were asked to rate their agreement with seventeen 5-point rating scale questions related to the usefulness and the usability of the toolkit and the workshop, with 1 indicating the most negative feedback and 5 indicating the most positive feedback. The 'usability’ part of the questionnaire design was inspired by the System Usability Scale 
(SUS) (Brooke 1996). Also there were two open-ended questions asking for the participants' comments on the toolkit design and the workshop.

Table 2 shows the key concepts of the seventeen questions and the corresponding ratings. The usefulness of toolkit was positively rated. Averaging the responses for all questions on usefulness (Q1 - Q8), the toolkit scored a mean of 3.61. The responses related to the usability of the toolkit were very positive. The mean value for all questions on usability of the toolkit and the workshop (Q9 - Q17) was 3.90 .

Other opinions from the participants were canvased from observation during the workshop and the responses to the open-ended questions. Comments that emerged were clustered into two themes.

Firstly, designing eco information individualisation was a complex concept. Both positive and negative comments were received on the effectiveness of the toolkit and the workshop to communicate this idea. It was observed that more experienced designers understood the concept more quickly and their design outputs were more developed compared to junior designers. Specific comments included:

‘...the complexity of the topic is massive and you made a good clarification and categorisation of all elements.'

'It has helped to give more insights into how the design process goes. [It] gives me new possibilities of using this in my design.'

'I enjoyed the workshop and found it thought provoking.'

'I like the diagram [the 3X3 matrix template].'

'I wished I understood the content much better.'

Secondly, more time was needed for some participants to understand the concept and digest the content of the design toolkit. Many participants expressed that they were confused during Task 1 (which was indeed purposefully set as an unguided activity), and would have preferred the toolkit to be introduced earlier. 
'Make the workshop more clear, explain better, and have less information. Too many information was displayed, and is not really clear the objective of the workshop.' 'I think more time needs to be provided for the first exercise because there is a lot of information to take in and to start ideating.'

' [The toolkit was] introduced too late in the process.'

\subsection{Discussions on workshop}

\subsubsection{Answering the research questions}

Through applying the toolkit in a design workshop and observing designers' performance in designing individualised labels, the three research questions set out at the beginning were addressed.

Can designers learn to design eco information individualisation in a short time? The design outputs from the workshop suggest that, with the aid of the design toolkit, it is possible for designers to learn the idea of eco information individualisation in a short time. The designers were able to generate a variety of ideas in the workshop, and demonstrated a progression on their design development after the design toolkit was introduced to them, although some individuals took longer to fully understand the framework and the design task than others.

Quality and feasibility of design outputs. Most of the design concepts generated were considered feasible by the workshop facilitator and have the potential to be developed into digital prototypes. To be suitable for digital prototype development, the design output should consist of a range of varied layouts in accordance to different user profiles. The label design should also indicate one or more eco attributes of the product (milk in this case). All examples shown in Figure 7 and Figure 9 satisfied these criteria.

How did the designers feel using the toolkit? Generally positive responses were received with regard to the usefulness and usability of the toolkit, despite some variability in the positiveness of the responses. The high ratio of cards that were marked as inspiring in Task 3 was a positive indication of 
the relevance of the card content. From observation during the workshop and the responses to the questionnaire, lessons were learnt for further improvement of the workshop and toolkit design.

\subsubsection{Limitations of the study}

The study presented in this paper was of a qualitative and explorative nature. A workshop can be used as a generative and an evaluative method. It has the strength of eliciting a rich picture of the experiences of the participants, but the reliability and validity of the data can be questionable. To increase the credibility of the outcomes, triangulation was done through using multiple data collection methods (observation, design outputs and questionnaire) as well as multiple data analysis methods (both quantitative and qualitative analysis of the questionnaire responses and the ideas generated). To increase transferability, this workshop had recruited both design students and designers with industrial experience.

Although improvements were observed in the design outputs generated after the toolkit was introduced, this could be due to a practice effect - the designers may have become more experienced and skilful over time. Other constraints of the study include the skills and interests of the participants, time constraints, and the artificial nature of the session.

This paper focuses on the feasibility of introducing the concept of eco information individualisation to designers in a short time with a design toolkit and a workshop. The design outputs from the workshop were reviewed based on two criteria: whether they represented the nature of eco information individualisation and their feasibility to be developed into working prototypes. To enhance objectivity, the design outputs were reviewed by the workshop main facilitator (one of the authors of this paper) and another author who was not involved directly in the workshop. However no other checking process by an independent researcher was carried out.

\section{Conclusions}

This paper reports on the development of a design research project investigating the opportunities provided by contextual technologies to individualise eco information design to encourage sustainable 
consumer behaviour.

A design toolkit was developed to extend the conceptual framework of eco information individualisation that we proposed in an earlier paper (Kwok, Harrison, and Qin 2014), in the hope of supporting problem framing and encouraging creativity during the design process for such a system. The toolkit contains sets of cards that delineate contexts related to user, product and relevant technologies, as we believe the attempts to create solutions evolve in parallel with the understanding of the problem. However we do not claim the cards are exhaustive. More cards can be added or renewed, especially over time when technologies continue to advance.

The toolkit was applied in a design workshop with designers. Positive responses were received with regard to its usefulness and usability. Results from the workshop show that it is possible for designers to learn to design eco information individualisation in a short time. The design outputs generated were considered largely feasible by the workshop facilitator.

This paper focuses on reporting the development and the usage of the toolkit and the workshop experiences, and is believed to be a step towards a greater understanding of designing eco information individualisation. Future studies will be conducted to evaluate the quality of the design outputs from the workshop. To further investigate the feasibility of the concept, we will also develop digital prototypes based on the design outputs generated in the workshop.

(Word count: 4496 words)

\section{References}

van Amstel, Mariëtte, Peter Driessen, and Pieter Glasbergen. 2008. "Eco-Labeling and Information Asymmetry: A Comparison of Five Eco-Labels in the Netherlands.” Journal of Cleaner Production 16(3): 263-76. http://linkinghub.elsevier.com/retrieve/pii/S0959652606002794 (January 7, 2014).

Arup. 2009. Drivers of Change. London: Prestel.

Bhamra, T, D Lilley, and T Tang. 2011. "Design for Sustainable Behaviour: Using Products to Change Consumer Behaviour.” The Design Journal 14(4): 427-45.

Brooke, John. 1996. “SUS: A Quick and Dirty Usability Scale.” In Usability Evaluation In Industry, eds. Patrick W. Jordan, Bruce Thomas, Bernard A. Weerdmeester, and Ian L. McClelland. , 189-94. 
Cohen, Mark a., and Michael P. Vandenbergh. 2012. "The Potential Role of Carbon Labeling in a Green Economy.” Energy Economics 34: S53-63.

http://linkinghub.elsevier.com/retrieve/pii/S0140988312002022 (December 20, 2013).

Froehlich, J. 2011. "Sensing and Feedback of Everyday Activities to Promote Environmental

Behaviors.” PhD diss.,University of Washington.

Hartikainen, Hanna, Taneli Roininen, Juha-Matti Katajajuuri, and Hannele Pulkkinen. 2013. "Finnish Consumer Perceptions of Carbon Footprints and Carbon Labelling of Food Products.” Journal of Cleaner Production. http://linkinghub.elsevier.com/retrieve/pii/S0959652613006215 (December 22, 2013).

IDEO. 2003. IDEO Method Cards: 51 Ways to Inspire Design. IDEO.

IDEO. 2015. The Field Guide to Human-Centered Design. 1st ed. San Francisco: IDEO.org.

Kofod-Petersen, A, and Agnar Aamodt. 2003. "Case-Based Situation Assessment in a Mobile Context-Aware System.” Artificial Intelligence in Mobile Systems: 41-49.

Kwok, Sze Yin, David Harrison, and Shengfeng Qin. 2013. "Design for Sustainable Behaviour : Proposing a New Eco-Feedback Device Based on Augmented Reality Technology.” In Sustainable Innovation 2013: Towards Sustainable Product Design 18th International Conference, Epsom: University for the Creative Arts, 118-30.

Kwok, Sze Yin, David Harrison, and Shengfeng Qin. 2014. "Designing an Individualised Eco Information System: A Conceptual Framework.” In 19th DMI: Academic Design Management Conference: Design Management in an Era of Disruption, London.

Lockton, Dan, David Harrison, and Neville A. Stanton. 2010. "The Design with Intent Method: A Design Tool for Influencing User Behaviour.” Applied Ergonomics 41(3): 382-92. http://linkinghub.elsevier.com/retrieve/pii/S0003687009001136 (January 12, 2016).

Pei, Eujin. 2009. "Building a Common Language of Design Representations for Industrial Designers and Engineering Designers.” PhD diss.,Loughborough University.

RSSB (Rail Safety and Standards Board). 2008. Understanding Human Factors - a Guide for the Railway Industry.

Scoble, Robert, and Shel Israel. 2014. Age of Context: Mobile, Sensors, Data and the Future of Privacy. 1st ed. Patrick Brewster Press.

Thøgersen, John. 2002. “Promoting 'Green’ Consumer Behavior with Eco-Labels.” In New Tools for Environmental Protection: Education, Information, and Voluntary Measures, eds. Thomas Dietz and Paul C. Stern. Washington, DC: The National Academies Press, 83-104.

Upham, Paul, Leonie Dendler, and Mercedes Bleda. 2011. "Carbon Labelling of Grocery Products: Public Perceptions and Potential Emissions Reductions.” Journal of Cleaner Production 19(4): 348-55. http://linkinghub.elsevier.com/retrieve/pii/S0959652610002015 (December 21, 2013). 
Table 1. Summary of design toolkit card structure and card names

\begin{tabular}{|l|l|l|l|}
\hline Type of cards & Person Cards & Product Cards & Technology Cards \\
\hline Title of card & 1. Verbaliser vs Imager (VI) & 9. Product Category & 17. Barcode \& QR code \\
\cline { 2 - 4 } & 2. Wholist vs Analytic (WA) & 10. Issuing organisation & 18. Augmented Reality (AR) \\
\cline { 2 - 4 } & 3. Preference \& Interest & $\begin{array}{l}\text { 11. Life Cycle Analysis } \\
\text { (LCA) }\end{array}$ & 19. iBeacon \\
\cline { 2 - 4 } & 4. Physiological factors & 12. Eco attribute & 20. RfID \& NFC \\
\cline { 2 - 4 } & 5. Time \& Location & 13. Colour & 21. Mobile \& Wearables \\
\cline { 2 - 4 } & 6. Task Related & 14. Data visualisation & 22. Social Media \\
\cline { 2 - 4 } & 7. History \& habit & 15. Level of detail & 23. Internet of Things (IoT) \\
\cline { 2 - 4 } & 8. Social factors & 16. Richer medium & $\begin{array}{l}\text { 24. Location-based service } \\
\text { (LBS) }\end{array}$ \\
\cline { 2 - 4 } & & & 25. Cloud computing \\
\hline
\end{tabular}


Table 2. Feedback on the usability and the usefulness of the toolkit and workshop

\begin{tabular}{|c|c|c|c|c|c|c|c|c|}
\hline \multirow{2}{*}{$\begin{array}{l}\text { Aspect } \\
\text { Usefulness }\end{array}$} & \multirow{2}{*}{$\begin{array}{l}\text { Mean } \\
3.61 \\
\end{array}$} & \multicolumn{2}{|c|}{ Key concept of the question } & \multirow{2}{*}{$\begin{array}{l}\mathrm{N} \\
11 \\
\end{array}$} & \multirow{2}{*}{$\begin{array}{l}\text { Mean } \\
4.27\end{array}$} & \multirow{2}{*}{$\begin{array}{l}\text { Media } \\
\mathrm{n}\end{array}$} & \multirow{2}{*}{\begin{tabular}{|l|} 
Mode \\
4 \\
\end{tabular}} & \multirow{2}{*}{$\begin{array}{l}\text { Range } \\
2\end{array}$} \\
\hline & & Q1 & Text on card is informative & & & & & \\
\hline & & Q2 & Images on card are informative & 11 & 3.91 & 4 & 5 & 1 \\
\hline & & Q3 & Cards provide information needed & 11 & 3.68 & 4 & 4 & 3 \\
\hline & & Q4 & Understanding of eco info individualisation & 10 & 3.40 & 4 & 4 & 3 \\
\hline & & Q5 & Toolkit supports problem framing & 11 & 3.73 & 4 & 4 & 3 \\
\hline & & Q6 & Worksheets trigger ideas & 10 & 3.10 & 3 & 3 & 3 \\
\hline & & Q7 & Cards trigger ideas & 11 & 3.27 & 3 & 3 & 3 \\
\hline & & Q8 & Toolkit is useful in guiding design & 11 & 3.55 & 4 & 4 & 2 \\
\hline \multirow[t]{9}{*}{ Usability } & \multirow[t]{9}{*}{3.90} & Q9 & Card info is well structured & 11 & 3.73 & 4 & 3 & 2 \\
\hline & & Q10 & Text on card is clear & 10 & 4.10 & 5 & 5 & 3 \\
\hline & & Q11 & Card layout & 11 & 3.73 & 4 & 4 & 3 \\
\hline & & Q12 & Worksheet layout & 11 & 3.55 & 4 & 4 & 3 \\
\hline & & Q13 & Colour of cards & 10 & 4.40 & 5 & 5 & 2 \\
\hline & & Q14 & Readability of cards & 11 & 3.64 & 4 & 4 & 3 \\
\hline & & Q15 & Size of cards & 11 & 4.27 & 4 & 5 & 2 \\
\hline & & Q16 & Experience using toolkit & 11 & 3.64 & 4 & 4 & 2 \\
\hline & & Q17 & Experience attending workshop & 11 & 4.09 & 4 & 4 & 1 \\
\hline
\end{tabular}




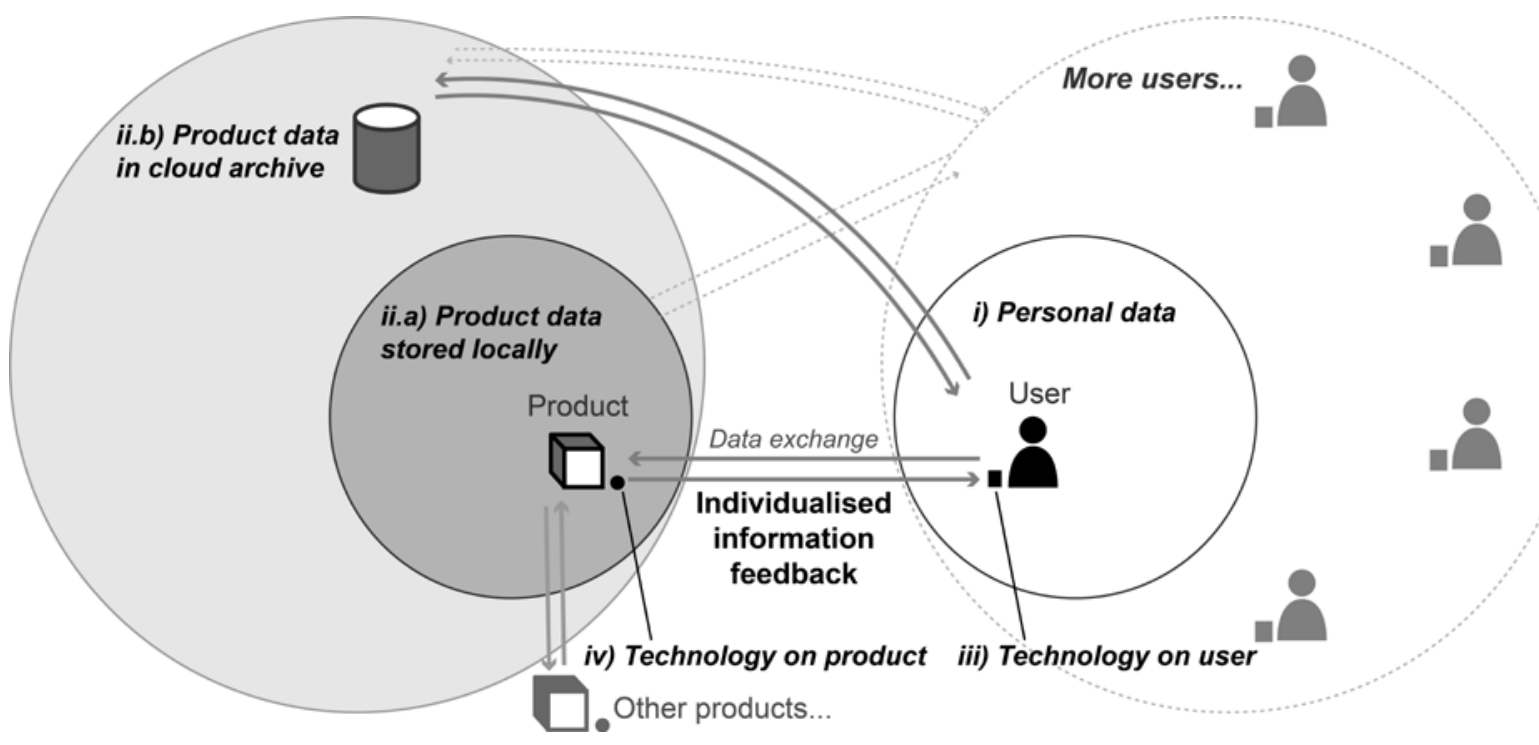

Figure 1. Refined conceptual framework of Eco Information Individualisation (Kwok, Harrison, and Qin 2014)

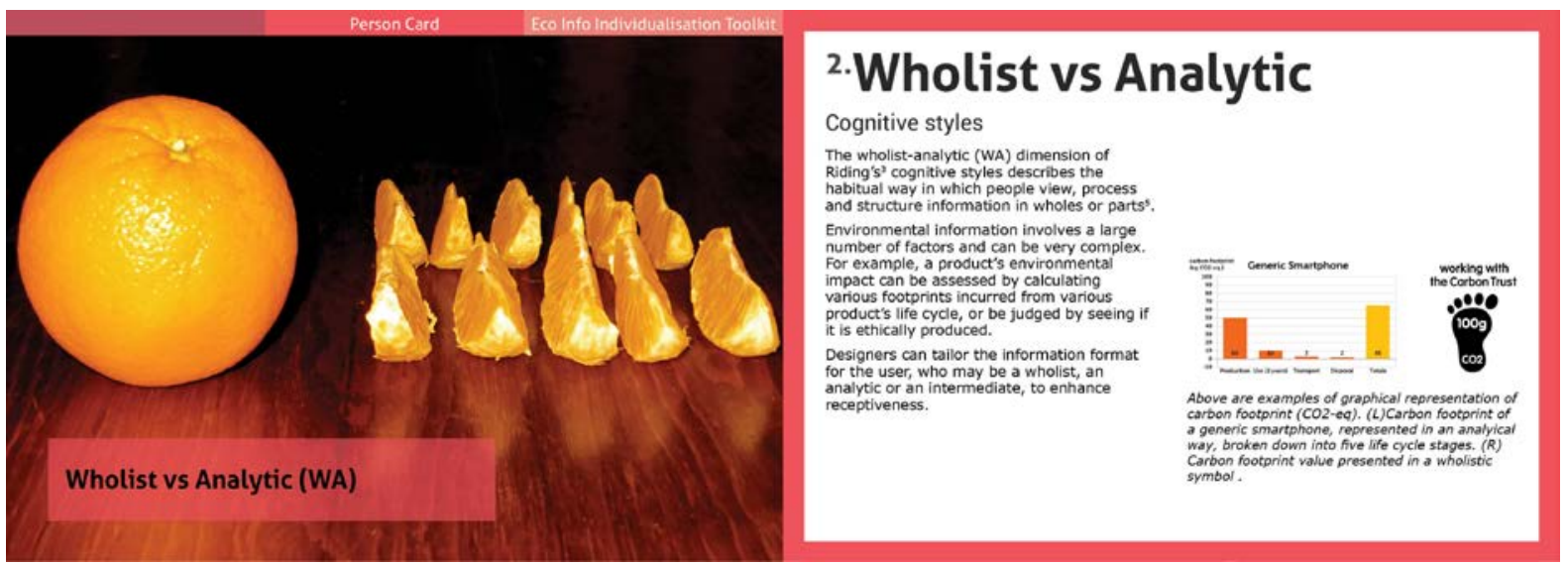

Figure 2. An example of the person card 
Individualised Eco Label

Tailoring upon 2 dimensions of 3 levels

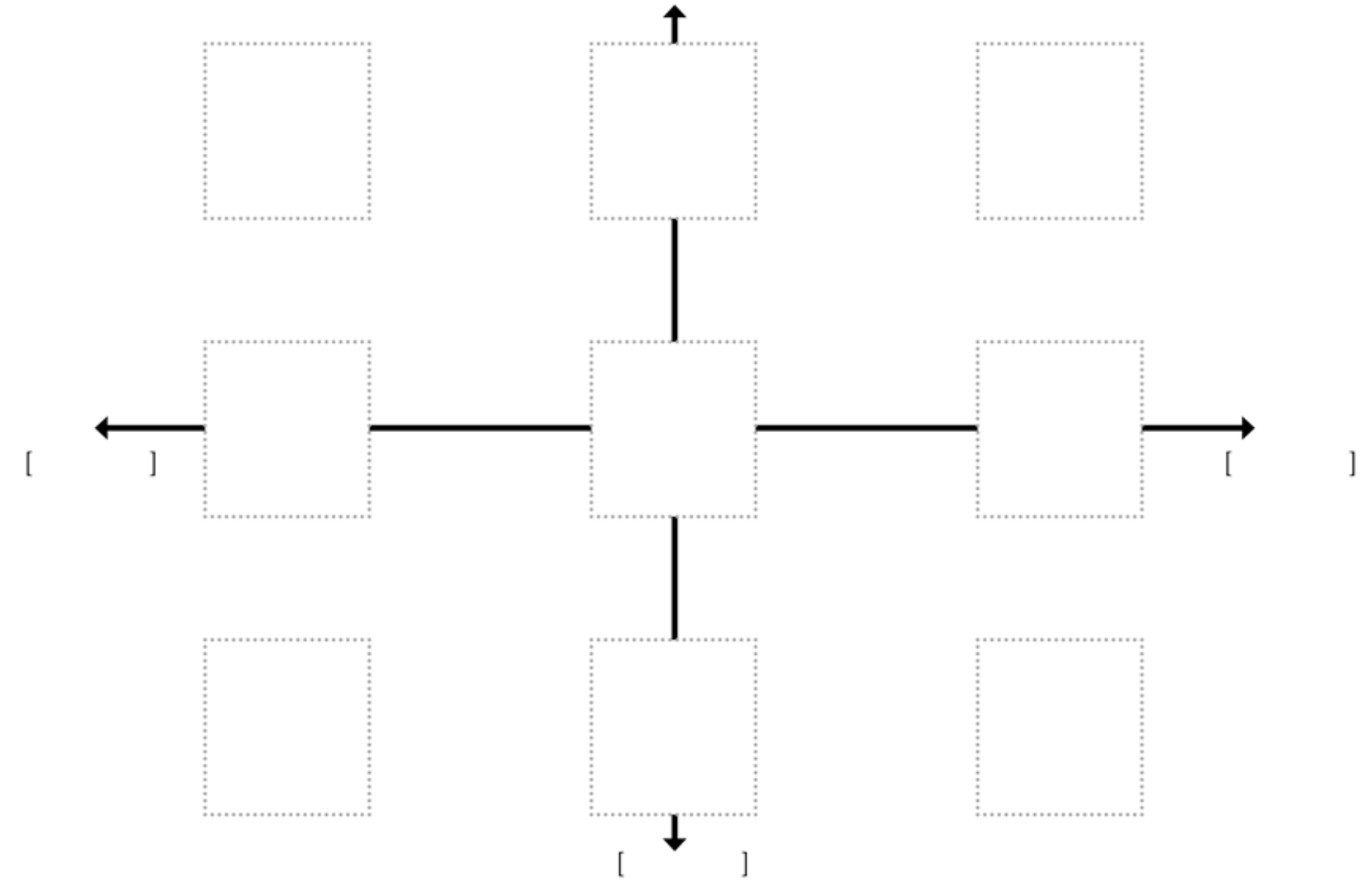

Figure 3. Image of $3 X 3$ matrix label design template. See Figure 7 and Figure 9 for examples of template usage. 


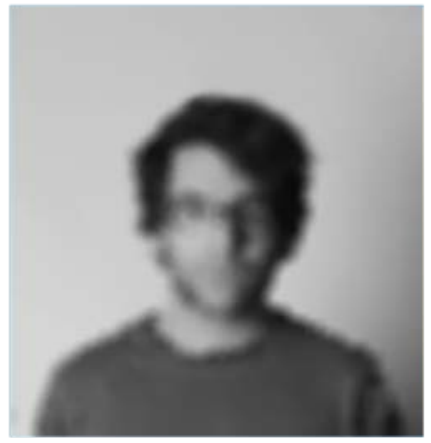

Figure 4. An example of the personas 

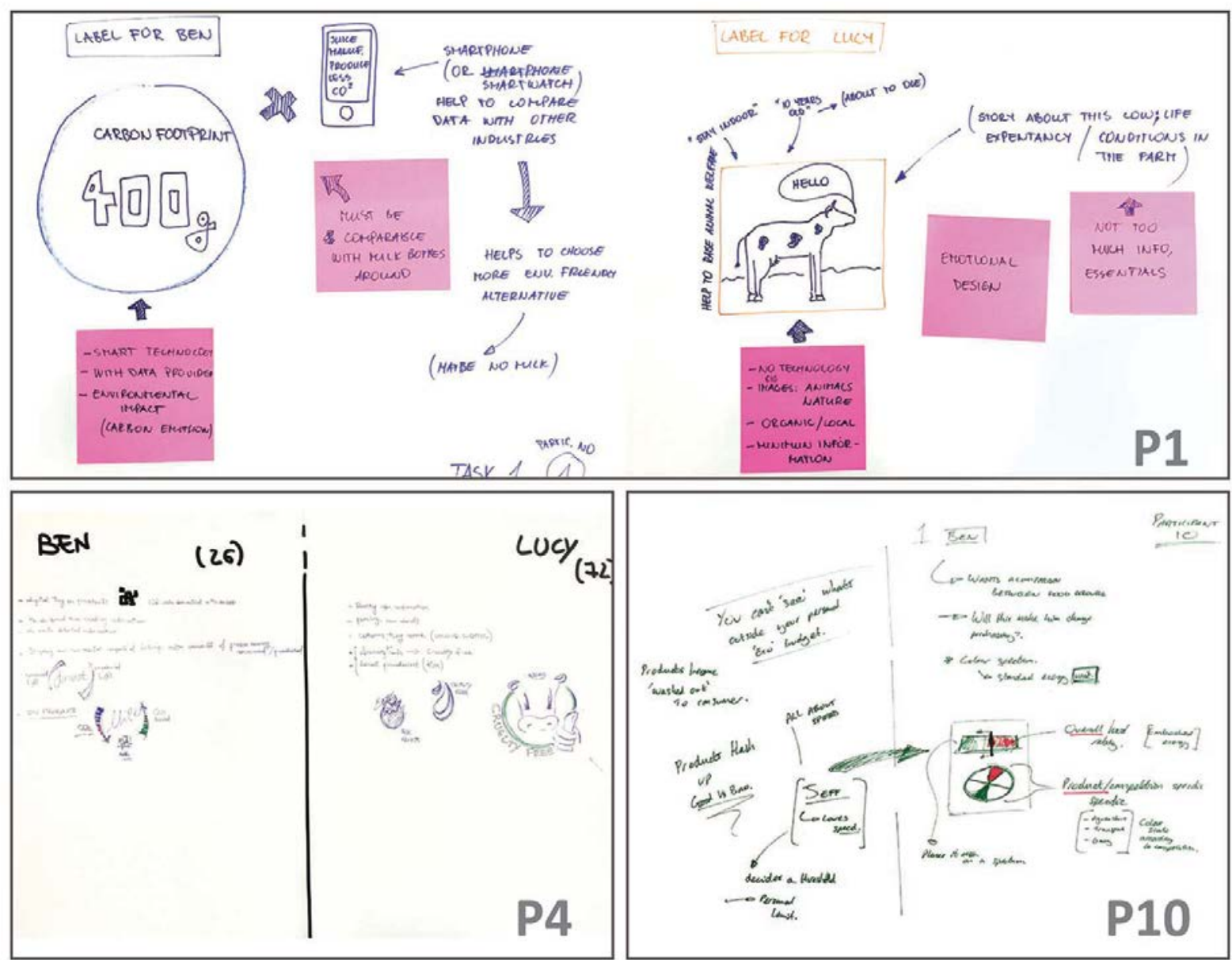

Figure 5. Selected design outcomes from Task 1 


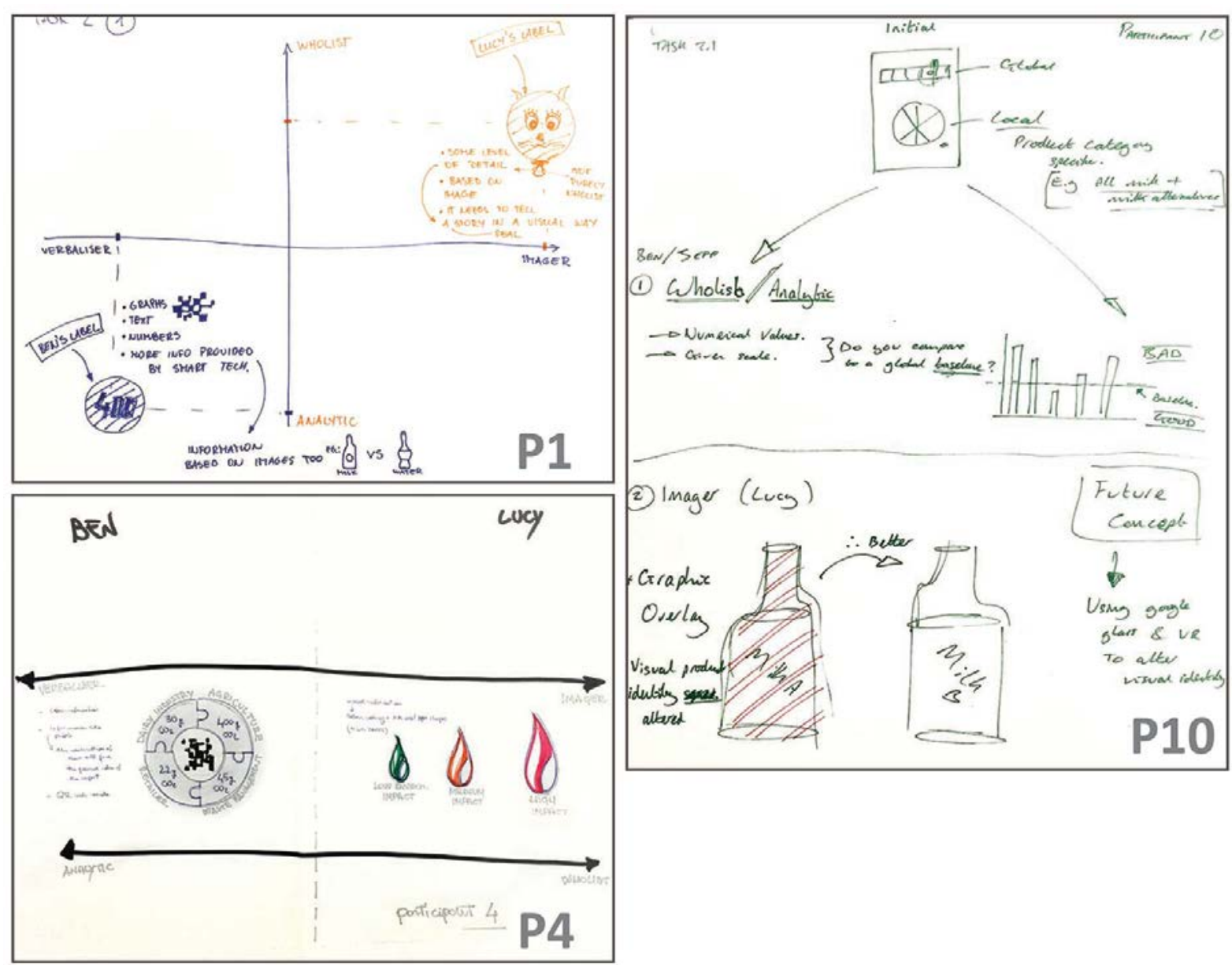

Figure 6. Selected design outcomes from Task 2.1 


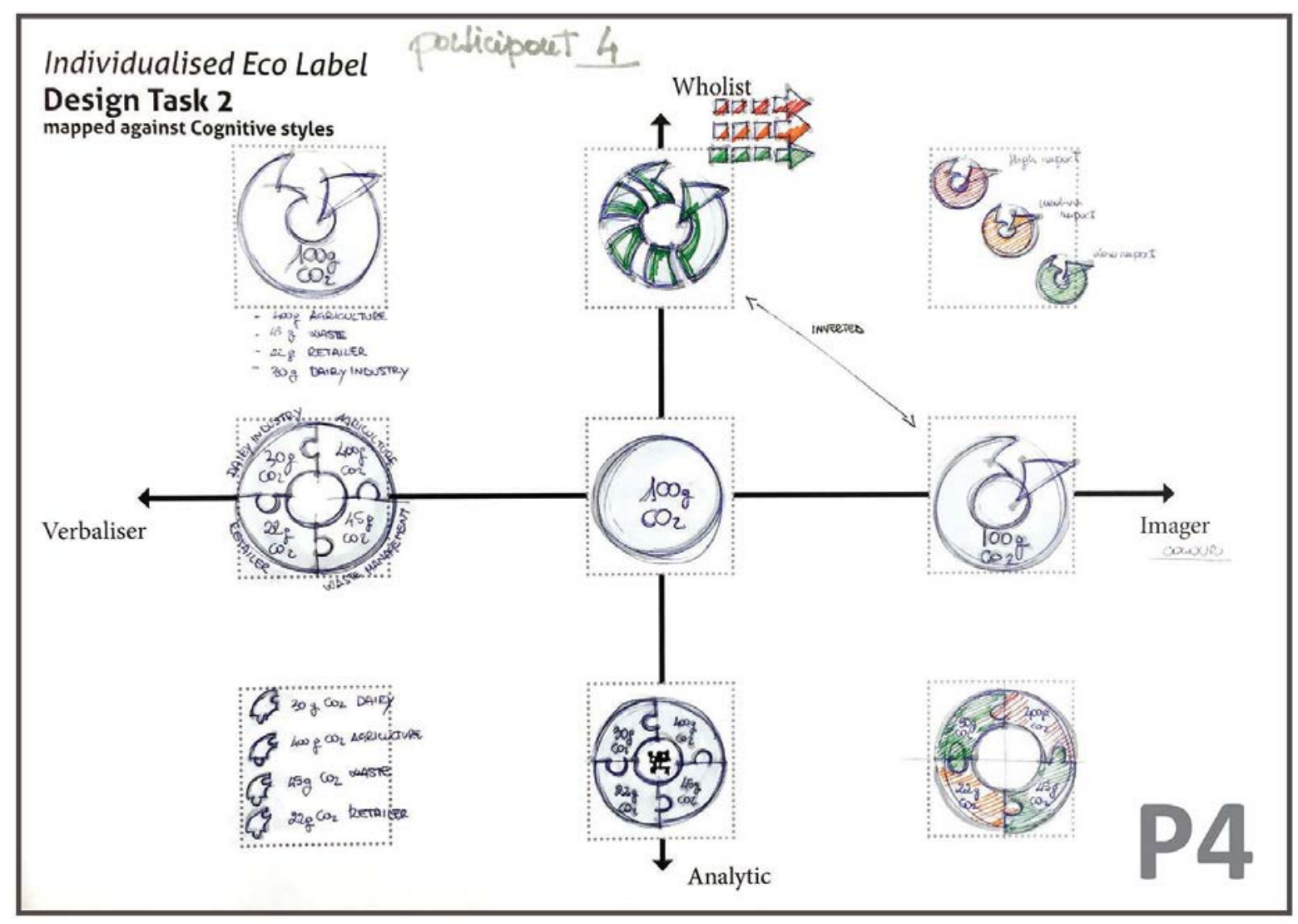

Figure 7. Selected design outcome from Task 2.2
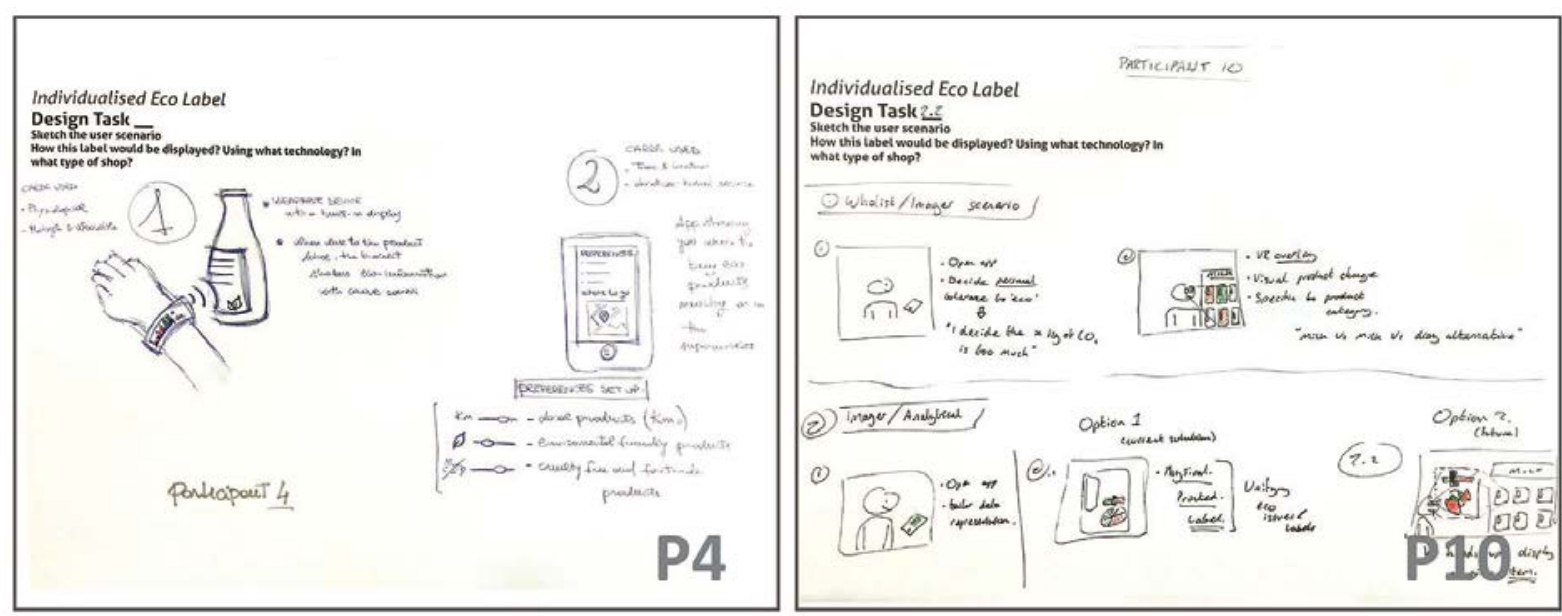

Figure 8. Selected sketches of user scenario 

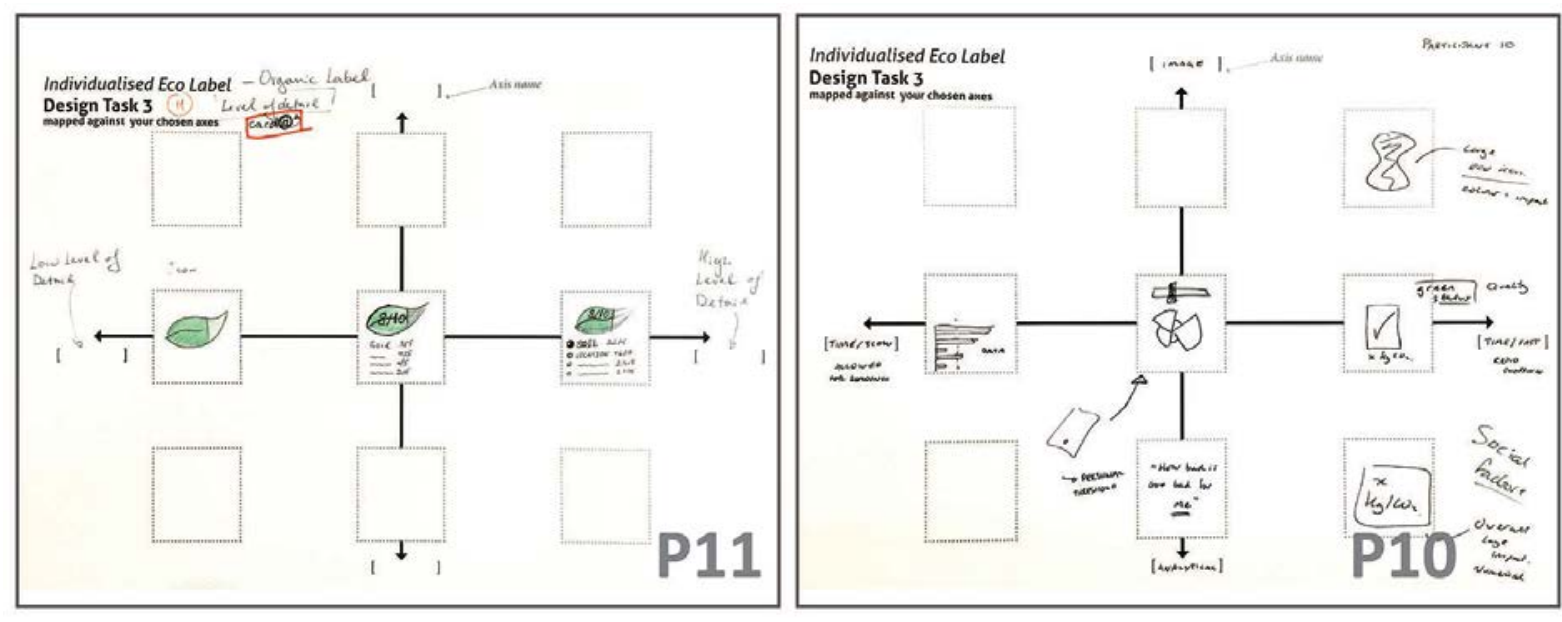

Figure 9. Selected design outcomes from Task 3

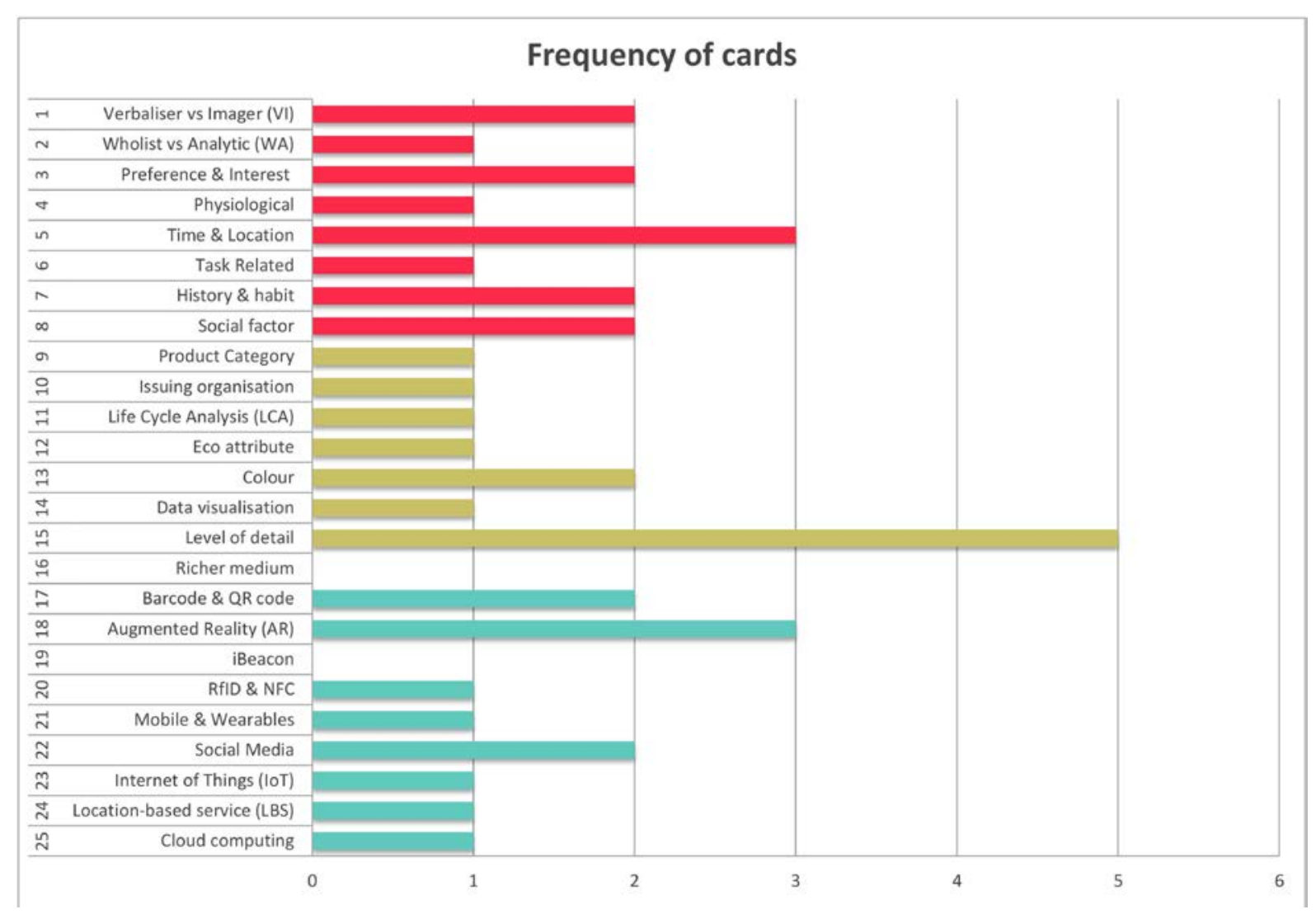

Figure 10. Summary of frequency of cards marked useful in triggering ideas 